\title{
Molecular architecture of the human caveolin-1 complex
}

Jason C. Porta ${ }^{1, \dagger}$, Bing Han ${ }^{2,3, \dagger}$, Alican Gulsevin ${ }^{4 \dagger}$ Jeongmin Chung ${ }^{1,6}$, Yelena Peskova ${ }^{2,3}$, Sarah Connolly ${ }^{1}$, Hassane S. Mchaourab ${ }^{7}$, Jens Meiler ${ }^{4,5}$, Erkan Karakas ${ }^{7, \S}$, Anne K. Kenworthy ${ }^{2,3, \S,}$ and Melanie D. Ohi ${ }^{1,8, \S}$

${ }^{1}$ Life Sciences Institute, University of Michigan, Ann Arbor, MI, USA.

${ }^{2}$ Center for Membrane and Cell Physiology, University of Virginia, Charlottesville, VA USA.

${ }^{3}$ Department of Molecular Physiology and Biological Physics, University of Virginia School of Medicine, Charlottesville, VA USA.

${ }^{4}$ Department of Chemistry, Vanderbilt University Nashville, TN, USA

${ }^{5}$ Institute for Drug Discovery, Leipzig University, Germany

${ }^{6}$ Department of Biotechnology, The Catholic University of Korea, Bucheon, Republic of Korea

${ }^{7}$ Department of Molecular Physiology and Biophysics, Vanderbilt University Nashville, TN, USA

${ }^{8}$ Department of Cell and Developmental Biology, University of Michigan School of Medicine, Ann Arbor, MI, USA

${ }^{\dagger}$ Co-first Authors

§Correspondence: erkan.karakas@vanderbilt.edu , akk7hp@virginia.edu, or mohi@umich.edu

One-Sentence Summary: Cryo-electron microscopy reveals that Caveolin-1 oligomerizes into a tightly packed disc with a flat membrane-binding surface. 


\begin{abstract}
:
Membrane sculpting proteins shape the morphology of cell membranes and facilitate remodeling in response to physiological and environmental cues. Complexes of the monotopic membrane protein caveolin function as essential curvature-generating components of caveolae, flask-shaped invaginations that sense and respond to plasma membrane tension. However, the structural basis for caveolin's membrane remodeling activity is currently unknown. Here, we show, using cryo-electron microscopy, that the human caveolin-1 complex is composed of 11 protomers organized into a tightly packed disc with a flat membrane-embedded surface. The structural insights suggest a new mechanism for how membrane sculpting proteins interact with membranes and reveal how key regions of caveolin-1, including its scaffolding, oligomerization, and intramembrane domains, contribute to its function.
\end{abstract}

\title{
Main Text:
}

Biological membranes assume a wide range of morphologies and are actively remodeled to support the normal physiological functions of cells as well as cell growth and differentiation (1, 2). An important mechanism for shaping and remodeling membranes is through the activity of curvature-inducing proteins (2-4). Caveolins, a family of monotopic membrane proteins first identified over 30 years ago, fall into this class of proteins based on their ability to bend membranes to form 50-100 $\mathrm{nm}$ flask shaped invaginations of the plasma membrane known as caveolae $(5,6)$. Comprising up to $30-50 \%$ of the surface area of some cell types (7), caveolae serve as mechanosensors and mechanoprotectors that sense and respond to changes in membrane tension at the cell surface by reversibly flattening ( 8 ). The absence of caveolin-1 (Cav1) and thus caveolae has profound consequences for mice, including decreased lifespan, lipid metabolism disorders, vascular abnormalities, dilated cardiomyopathy, and pulmonary hypertension (9). In humans, Cav1 has been linked to cardiovascular disease, cancer, lipodystrophy, and kidney disease (10-15). How Cav1 facilitates caveolae biogenesis is not fully understood, but is thought to require the insertion of a highly hydrophobic region of the protein, termed the intramembrane domain (IMD), into the cytoplasmic face of the plasma membrane to induce membrane curvature via a wedging mechanism $(3,4,16,17)$. To induce curvature, Cav1 must also assemble correctly into oligomeric complexes $8 \mathrm{~S}$ in size that undergo additional higher order interactions with each other and the cavin proteins to form mature caveolae that are polygonal in shape (18-21). 8S complex formation occurs via a cooperative process mediated by its oligomerization domain (OD) and aided by its scaffolding domain (SD) and signature motif (SM) (21-23). However, the structural basis for Cav1's membrane bending activity remains unknown, as only low-resolution structures of caveolin complexes currently exist (24-26).

Although caveolin proteins are found exclusively in metazoans (18), when expressed in Escherichia coli, Cav1 forms 8S-like complexes (26) and induces the formation of heterologous caveolae (h-caveolae) $(27,28)$. Thus, E. coli-expressed Cav1 forms oligomers and sculpts membranes, two of its essential functions in mammalian cells. Using single-particle cryo-EM analysis, we determined the structure of E. coli expressed and purified complexes (26) (Fig. 1, Figs. S1-3, and Methods). 2D class averages of en face views of the complex showed 11 spiraled $\alpha$-helices, and an ab initio 3D reconstruction with no enforced symmetry resulted in a low-resolution map with structural features indicating potential 11-fold symmetry (Fig. S3C, D). Applying C11 symmetry during 3D refinement steps led to a $3.5 \AA$ resolution structure (Fig. 1 and Fig. S4). This agrees with previous reports suggesting 7-14 copies of Cav1 per 8 S complex $(21,22,26)$. The 11 Cav1 protomers organize into a disc-shaped complex with a diameter of $\sim 140 \AA$ and a height of $\sim 34 \AA$ (Fig. $1 \mathrm{~A}-\mathrm{F})$. To ensure we were not missing other oligomeric forms of the complex by enforcing 11-fold symmetry, we performed $3 \mathrm{D}$ refinements using 
symmetries spanning C8-C14. None of these resulted in maps with apparent secondary structural features or improved resolutions (Fig. S5). Additionally, 3D classification did not yield models of any other oligomeric forms.

The structure reveals Cav1 oligomerizes into a disc that contains an outer "rim" ( $23 \AA$ wide), a central $\beta$-barrel "hub" ( 28 Å wide), and eleven curved $\alpha$-helical "spokes". Each Cav1 protomer is mostly $\alpha$-helical ( $\alpha 1-5)$, consistent with previous reports (29) (Fig. 1D-H). Viewed from the side, helices $\alpha 2-\alpha 5$ of the protomer align along the flat surface of the complex, and helix 1 forms a $\sim 20^{\circ}$ angle to this surface (Fig. 1H). Cav1 also contains two non-helical regions located at both termini (Fig. 1D-H). The N-terminus, which makes up part of the "rim" region, consists of a loop with a short $3_{10}$ helix $(\eta 1)$ (Fig $\left.1 G, H\right)$. This region makes a $180^{\circ}$ turn at the outer edge of the complex, intercalating with the spokes and $\mathrm{N}$-terminal regions of neighboring protomers (Fig. 1A-H). Each protomer terminates in a C-terminal $\beta$-strand angled by $\sim 40^{\circ}$ relative to the plane of the flat surface of the disc. These $\beta$-strands interact to form an 11-stranded parallel $\beta$ barrel "hub" (Fig. 1A-H). Based on the position of the detergent micelle in the density map (Fig. 1I) and our previous negative stain analysis of $h$-caveolae (26), the flat face of the complex corresponds to the membrane-facing surface, whereas the $\beta$-barrel faces the cytoplasm. Strikingly, the central cavity of the barrel extends entirely through the complex and is open to solvent at the cytoplasmic-facing side (Fig. 1A-F and $1 \mathrm{I}$ ). The detergent micelle covers $\sim 60 \%$ of the surface area of the $8 S$ complex, including the membrane facing surface, and extends $\sim 14 \AA$ around the sides of the outer rim of the disc (Fig. 1I).

From the density map, we built an atomic model for most of Cav1 (residues 49-177) (Fig. 2A-D). However, residues 1-48, which fall within a predicted disordered region $(28,29)$ and are dispensable for caveolae biogenesis (18), were not detected in the map (Fig. 2A-D and Fig. S6). To correlate the extensive biochemical and functional data in the literature with our structural analysis, we mapped key regions and residues of Cav1 onto the structure (Fig. 2A-G, Fig. S7, and Supplementary Video 1). The OD (residues 61-101) (22) is located at the outer rim of the disc and contributes to extensive subunit interfaces. The SM (residues 68-75) $(30,31)$ and SD (residues 82-101) (32) both fall within the OD. Lying within the loop region, the SM forms tight contacts with two neighboring protomers, whereas the SD comprises most of helix $\alpha 1$ and encircles the periphery of the complex. The residues separating the SM and SD make two $90^{\circ}$ turns, bringing these two motifs into proximity (Fig. 2D). The IMD (residues 102-134) $(23,29)$ begins on the $\mathrm{C}$-terminal end of the helix $\alpha 1$ immediately adjacent to the SD and continues across helices $\alpha 2$, $\alpha 1$, and part of $\alpha 3$ (Fig. 2A-D). Known phosphorylation and ubiquitination (33, 34) sites are accessible on the cytoplasmic face of the complex, whereas Cav1's palmitoylation sites (Cys133, Cys143, and Cys156) (35) are located on the membrane-facing surface (Fig. S8).

In addition to these previously characterized functional regions, we identified three new motifs in Cav1 that are critical for $8 S$ complex stability. First, residues 49-60 form a loop extending over the SM of the neighboring protomer (Figs. 2A-D and 3 ). This previously unnamed region is required for caveolae assembly (18) and localization in migrating fibroblasts (36). The structure shows that these residues appear to "lock" the interaction between protomers, leading us to designate this region as the "pin motif" (PM) (Fig. 2A-G and 3; Fig. S7). Second, residues 135169 , a region required for caveolae formation (18), oligomerization, and exit of the protein from the Golgi complex (20), generate a spoke region (SR) organized parallel to the membrane plane (Fig. 2A-D; Fig. S7). The structure shows these residues form the planar hydrophobic surface on the membrane-facing side of the complex while also creating a highly negatively charged surface on the cytoplasm-facing side of the complex (Fig. 2G, J). Helix a5 (residues 143-155) distorts this flat surface by bending towards the cytoplasm facing side of the complex where it connects to a $\beta$-strand (residues 170-176) (Figs. 1G, H and 2C, D). $\beta$-strands from adjacent protomers assemble into the third new motif, an eleven-stranded parallel $\beta$-barrel with a 
hydrophilic exterior and hydrophobic interior (Fig. 2A-G). To our knowledge, this represents the largest reported example of a parallel $\beta$-barrel to date; the most similar $\beta$-barrel reported in the PDB (2AO9) was observed in a soluble bacteriophage protein of unknown function and contains nine parallel $\beta$-strands (Fig. S9A). The barrel is capped by Lys176, introducing a highly positively charged layer separating the hydrophobic interior of the barrel from the cytoplasm. The narrowest accessible region of the interior of the $\beta$-barrel has a diameter of $15 \AA$ (Fig. S9B), making the channel large enough to accommodate small molecules such as lipids. However, no density was detected inside the $\beta$-barrel, perhaps because of the imposed $C 11$ symmetry.

The structure also unveils atomic details of how protomers oligomerize to form 8S complexes, a process essential for caveolae biogenesis. Classically, the OD has been proposed to function as the main region driving protomer-protomer interactions that lead to $8 \mathrm{~S}$ complex formation (9). The structure reveals that instead extensive networks of interactions occur along the entirety of each protomer, including the rim, spoke, and hub regions (Fig. 3 and Fig. S9C-F). Starting at the "rim", the N-terminus of one protomer ( $i)$ engages four neighboring protomers $(i-2, i-1, i+1$, and i+2) (Fig. 3A, B). The ODs from neighboring protomers are arranged adjacent to each other, but only slightly overlap (Fig. 3B). Instead, the OD of protomer $i$ stacks onto the cytoplasmic side of the IMDs of the neighboring protomers $i+1$ and $i+2$ (Fig. 3A, B). Within $\mathrm{OD}_{i}, \mathrm{SM}_{i}$ is sandwiched between $\alpha 1_{i+1}, \alpha 2_{i+1}, \alpha 2_{i+2}, \alpha 3_{i+2}, \pi 1_{i+2}$ and $\mathrm{PM}_{i+1}$. Arg5 $4_{i+1}$ of $\mathrm{PM}_{i+1}$ intercalates into a pocket formed by His79 $i$ and Trp85, pinning $\mathrm{OD}_{i}$ in place (Fig. 3A,B). To test the importance of this newly described molecular "pin", we mutated Arg54, a residue predicted to play an important role in facilitating protomer-protomer interactions, to alanine. This mutation severely distrupts the $8 \mathrm{~S}$ complexes, confirming that the PM interaction with the SM of the neighboring protomer is important for the $8 \mathrm{~S}$ complex formation (Fig. S10). The highly hydrophobic IMD helices ( $\alpha 2, \pi 1$, and part of $\alpha 3$ ) lay underneath the OD of the neighboring protomers, contributing to oligomerization (Fig. 3A, C). $\alpha 2_{i+2}$ forms a helical bundle that crosses $\alpha 1_{i+1}$ facilitated by the small side chains of residues Ala112 $2_{i+1}$, Gly116 $6_{i+1}$, and Ala120 ${ }_{i+1}$. The side chain of Trp $128_{i+2}$ extends from $\pi 1_{i+2}$ into the cavity formed by $O D_{i}$ and the loop between $\alpha 1_{i+1}$ and $\alpha 2_{i+1}$, suggesting it plays a critical role in oligomerization (Fig. 3A, C). The remaining helices forming the SR (C-terminal portion of $\alpha 3, \alpha 4$, and $\alpha 5$ ) make pairwise contacts across protomers (Fig. 3C, Fig. S9C-F). These interactions involve residues with larger side chains, increasing the separation of the helices compared to those of the $\alpha 1_{i}-\alpha 2_{i+1}$ crossing, which ultimately converge to form the $\mathrm{C}$-terminal $\beta$-barrel, the final major region of protomer-protomer interactions. The symmetry dictates a two-residue offset between neighboring protomers, creating a $\beta$-barrel (11, 22). Interestingly, several disease-associated mutations in Cav1 localize on the connecting regions between the IMD and SR, or the SR and $\beta$-barrel (Fig. S8), suggesting that this region of the complex is sensitive to mutations that destabilize the complex $(14,15)$.

The structure of the human Cav1 8S complex provides a molecular framework for understanding the overall organization of Cav1 oligomers, the exact roles the OD, SD, SM, and IMD play in complex formation, the importance of previously unrecognized regions of the protein, and the impact of disease-associated mutations such as $\mathrm{P} 132 \mathrm{~L}$ (12) (Fig. S8H, I) on the structure. Importantly, it also suggests new models for how Cav1 packs in membranes and controls caveolae architecture (Fig. S11). In contrast to the prevailing model suggesting the IMD forms a hairpin-like structure that inserts into the membrane creating a wedge that bends membrane $(3,4,16,17)$, the structure of the $8 \mathrm{~S}$ Cav1 complex reveals that the IMD contributes to the formation of a flat membrane-facing surface while simultaneously stabilizing contacts between protomers. The outside of the outer rim of the complex is also primarily hydrophobic. Together, these features suggest a model in which the membrane-associated side of the complex embeds deeply within the cytoplasmic leaflet, interacting with the terminal carbons of the lipids of the opposing leaflet rather than sitting at the interface between the headgroups and acyl chains as typically would be expected for amphipathic helices (Fig. 4B). In this model, the $8 \mathrm{~S}$ complex, by displacing lipids from the cytoplasmic leaflet, could create an ordered 
membrane nanodomain composed of protein on one membrane leaflet and lipids on the other. Interestingly, a single $8 \mathrm{~S}$ complex fits snugly on each face of a dodecahedron of the characteristic size of $h$-caveolae (30 nm average diameter) (28) (Fig. S11a), whereas up to three can be accommodated on each surface of a mammalian caveolae $(61 \mathrm{~nm}$ average diameter) assuming dodecahedral symmetry (25) (Fig. S11B-C). Thus, in caveolae, caveolin complexes may function primarily by stabilizing flat membrane surfaces of polyhedral structures rather than imposing continuous membrane curvature, defining a new mechanism for how integral membrane proteins sculpt cell membranes to form functional domains.

\section{References}

1. J. C. Bozelli, Jr., R. M. Epand, Membrane Shape and the Regulation of Biological Processes. J Mol Biol 432, 5124-5136 (2020).

2. K. J. Day, J. C. Stachowiak, Biophysical forces in membrane bending and traffic. Curr Opin Cell Biol 65, 72-77 (2020).

3. I. K. Jarsch, F. Daste, J. L. Gallop, Membrane curvature in cell biology: An integration of molecular mechanisms. J Cell Biol 214, 375-387 (2016).

4. C. Has, S. L. Das, Recent developments in membrane curvature sensing and induction by proteins. Biochim Biophys Acta Gen Subj 1865, 129971 (2021).

5. K. G. Rothberg et al., Caveolin, a protein component of caveolae membrane coats. Cell 68, 673-682 (1992).

6. J. R. Glenney, Jr., D. Soppet, Sequence and expression of caveolin, a protein component of caveolae plasma membrane domains phosphorylated on tyrosine in Rous sarcoma virus-transformed fibroblasts. Proc Natl Acad Sci U S A 89, 10517-10521 (1992).

7. H. Thorn et al., Cell surface orifices of caveolae and localization of caveolin to the necks of caveolae in adipocytes. Mol Biol Cell 14, 3967-3976 (2003).

8. R. G. Parton, M. A. del Pozo, Caveolae as plasma membrane sensors, protectors and organizers. Nat Rev Mol Cell Biol 14, 98-112 (2013).

9. I. Mercier et al., Clinical and translational implications of the caveolin gene family: lessons from mouse models and human genetic disorders. Lab Invest 89, 614-623 (2009).

10. L. Shu et al., Shared genetic regulatory networks for cardiovascular disease and type 2 diabetes in multiple populations of diverse ethnicities in the United States. PLoS Genet 13, e1007040 (2017).

11. C. A. Kim et al., Association of a homozygous nonsense caveolin-1 mutation with Berardinelli-Seip congenital lipodystrophy. J Clin Endocrinol Metab 93, 1129-1134 (2008).

12. G. Bonuccelli et al., Caveolin-1 (P132L), a common breast cancer mutation, confers mammary cell invasiveness and defines a novel stem cell/metastasis-associated gene signature. Am J Pathol 174, 1650-1662 (2009).

13. E. D. Austin et al., Whole exome sequencing to identify a novel gene (caveolin-1) associated with human pulmonary arterial hypertension. Circ Cardiovasc Genet 5, 336343 (2012).

14. B. Han et al., Characterization of a caveolin-1 mutation associated with both pulmonary arterial hypertension and congenital generalized lipodystrophy. Traffic 17, 1297-1312 (2016).

15. C. A. Copeland et al., A disease-associated frameshift mutation in caveolin-1 disrupts caveolae formation and function through introduction of a de novo ER retention signal. Mol Biol Cell 28, 3095-3111 (2017). 
16. J. Lee, K. J. Glover, The transmembrane domain of caveolin-1 exhibits a helix-breakhelix structure. Biochim Biophys Acta 1818, 1158-1164 (2012).

17. R. G. Parton, V. Tillu, K. A. McMahon, B. M. Collins, Key phases in the formation of caveolae. Curr Opin Cell Biol 71, 7-14 (2021).

18. M. Kirkham et al., Evolutionary analysis and molecular dissection of caveola biogenesis. J Cell Sci 121, 2075-2086 (2008).

19. X. Ren et al., Conformational defects slow Golgi exit, block oligomerization, and reduce raft affinity of caveolin-1 mutant proteins. Mol Biol Cell 15, 4556-4567 (2004).

20. T. Machleidt, W. P. Li, P. Liu, R. G. Anderson, Multiple domains in caveolin-1 control its intracellular traffic. J. Cell Biol. 148, 17-28 (2000).

21. A. Hayer, M. Stoeber, C. Bissig, A. Helenius, Biogenesis of caveolae: stepwise assembly of large caveolin and cavin complexes. Traffic 11, 361-382 (2010).

22. M. Sargiacomo et al., Oligomeric structure of caveolin: implications for caveolae membrane organization. Proc Natl Acad Sci U S A 92, 9407-9411 (1995).

23. S. Monier et al., VIP21-caveolin, a membrane protein constituent of the caveolar coat, oligomerizes in vivo and in vitro. Mol. Biol. Cell 6, 911-927 (1995).

24. G. Whiteley, R. F. Collins, A. Kitmitto, Characterization of the molecular architecture of human caveolin-3 and interaction with the skeletal muscle ryanodine receptor. $J$ Biol Chem 287, 40302-40316 (2012).

25. M. Stoeber et al., Model for the architecture of caveolae based on a flexible, net-like assembly of Cavin1 and Caveolin discs. Proc Natl Acad Sci U S A 113, E8069-E8078 (2016).

26. B. Han et al., Structure and assembly of CAV1 8 S complexes revealed by single particle electron microscopy. Sci Adv 6, (2020).

27. P. J. Walser et al., Constitutive formation of caveolae in a bacterium. Cell 150, 752-763 (2012).

28. N. Ariotti et al., Molecular characterization of caveolin-induced membrane curvature. J Biol Chem 290, 24875-24890 (2015).

29. K. T. Root, J. A. Julien, K. J. Glover, Secondary structure of caveolins: a mini review. Biochem Soc Trans 47, 1489-1498 (2019).

30. P. E. Scherer et al., Identification, sequence, and expression of caveolin-2 defines a caveolin gene family. Proc Natl Acad Sci U S A 93, 131-135 (1996).

31. Z. Tang et al., Molecular cloning of caveolin-3, a novel member of the caveolin gene family expressed predominantly in muscle. J Biol Chem 271, 2255-2261 (1996).

32. S. Li et al., Evidence for a regulated interaction between heterotrimeric $\mathrm{G}$ proteins and caveolin. J Biol Chem 270, 15693-15701 (1995).

33. A. R. Busija, H. H. Patel, P. A. Insel, Caveolins and cavins in the trafficking, maturation, and degradation of caveolae: implications for cell physiology. Am J Physiol Cell Physiol 312, C459-C477 (2017).

34. A. Schlegel, P. Arvan, M. P. Lisanti, Caveolin-1 binding to endoplasmic reticulum membranes and entry into the regulated secretory pathway are regulated by serine phosphorylation. Protein sorting at the level of the endoplasmic reticulum. J Biol Chem 276, 4398-4408 (2001).

35. D. J. Dietzen, W. R. Hastings, D. M. Lublin, Caveolin is palmitoylated on multiple cysteine residues. Palmitoylation is not necessary for localization of caveolin to caveolae. J. Biol. Chem. 270, 6838-6842 (1995).

36. X. H. Sun et al., Identification of a novel domain at the $\mathrm{N}$ terminus of caveolin-1 that controls rear polarization of the protein and caveolae formation. $J$ Biol Chem 282, 72327241 (2007).

37. F. W. Studier, Protein production by auto-induction in high density shaking cultures. Protein Expr Purif 41, 207-234 (2005). 
38. M. Ohi, Y. Li, Y. Cheng, T. Walz, Negative staining and image classification - powerful tools in modern electron microscopy. Biol Proced Online 6, 23-34 (2004).

39. C. Suloway et al., Automated molecular microscopy: the new Leginon system. $J$ Struct Biol 151, 41-60 (2005).

40. A. Punjani, J. L. Rubinstein, D. J. Fleet, M. A. Brubaker, cryoSPARC: algorithms for rapid unsupervised cryo-EM structure determination. Nat Methods 14, 290-296 (2017).

41. T. Bepler, K. Kelley, A. J. Noble, B. Berger, Topaz-Denoise: general deep denoising models for cryoEM and cryoET. Nat Commun 11, 5208 (2020).

42. J. Pfab, N. M. Phan, D. Si, DeepTracer for fast de novo cryo-EM protein structure modeling and special studies on CoV-related complexes. Proc Natl Acad Sci U S A 118, (2021).

43. R. Y. Wang et al., Automated structure refinement of macromolecular assemblies from cryo-EM maps using Rosetta. Elife 5, (2016).

44. M. D. Tyka et al., Alternate states of proteins revealed by detailed energy landscape mapping. J Mol Biol 405, 607-618 (2011).

45. B. Frenz, A. C. Walls, E. H. Egelman, D. Veesler, F. DiMaio, RosettaES: a sampling strategy enabling automated interpretation of difficult cryo-EM maps. Nat Methods, (2017).

46. P. Emsley, B. Lohkamp, W. G. Scott, K. Cowtan, Features and development of Coot. Acta Crystallogr D Biol Crystallogr 66, 486-501 (2010).

47. P. V. Afonine et al., Real-space refinement in PHENIX for cryo-EM and crystallography. Acta Crystallogr D Struct Biol 74, 531-544 (2018).

48. F. DiMaio, M. D. Tyka, M. L. Baker, W. Chiu, D. Baker, Refinement of protein structures into low-resolution density maps using rosetta. J Mol Biol 392, 181-190 (2009).

49. C. J. Williams et al., MolProbity: More and better reference data for improved all-atom structure validation. Protein Sci 27, 293-315 (2018).

50. A. Morin et al., Collaboration gets the most out of software. Elife 2, e01456 (2013).

51. E. F. Pettersen et al., UCSF Chimera--a visualization system for exploratory research and analysis. J Comput Chem 25, 1605-1612 (2004).

52. E. F. Pettersen et al., UCSF ChimeraX: Structure visualization for researchers, educators, and developers. Protein Sci 30, 70-82 (2021).

53. E. Krissinel, K. Henrick, Secondary-structure matching (SSM), a new tool for fast protein structure alignment in three dimensions. Acta Crystallogr D Biol Crystallogr 60, 22562268 (2004).

54. L. Holm, DALI and the persistence of protein shape. Protein Sci 29, 128-140 (2020).

55. R. Ayoub, Y. Lee, RUPEE: A fast and accurate purely geometric protein structure search. PLoS One 14, e0213712 (2019).

56. Z. Li, L. Jaroszewski, M. Iyer, M. Sedova, A. Godzik, FATCAT 2.0: towards a better understanding of the structural diversity of proteins. Nucleic Acids Res 48, W60-W64 (2020).

57. M. A. Lomize, I. D. Pogozheva, H. Joo, H. I. Mosberg, A. L. Lomize, OPM database and PPM web server: resources for positioning of proteins in membranes. Nucleic Acids Res 40, D370-376 (2012).

58. S. H. Lee, D. B. Hayes, G. Rebowski, I. Tardieux, R. Dominguez, Toxofilin from Toxoplasma gondii forms a ternary complex with an antiparallel actin dimer. Proc Natl Acad Sci U S A 104, 16122-16127 (2007).

59. Y. M. Abbas, D. Wu, S. A. Bueler, C. V. Robinson, J. L. Rubinstein, Structure of VATPase from the mammalian brain. Science 367, 1240-1246 (2020).

60. A. Andreeva, D. Howorth, C. Chothia, E. Kulesha, A. G. Murzin, SCOP2 prototype: a new approach to protein structure mining. Nucleic Acids Res 42, D310-314 (2014). 
61. A. Andreeva, E. Kulesha, J. Gough, A. G. Murzin, The SCOP database in 2020: expanded classification of representative family and superfamily domains of known protein structures. Nucleic Acids Res 48, D376-D382 (2020).

62. G. Klesse, S. Rao, M. S. P. Sansom, S. J. Tucker, CHAP: A Versatile Tool for the Structural and Functional Annotation of Ion Channel Pores. J Mol Biol 431, 3353-3365 (2019).

Acknowledgments: We thank Dr. K. Jebrell Glover for providing cDNA constructs and Drs. Jochen Zimmer, Charles Sanders, James Casanova, and Ludger Johannes for feedback on the manuscript. The University of Michigan Cryo-EM Facility (U-M Cryo-EM) has received generous support from the U-M Life Sciences Institute and the U-M Biosciences Initiative.

\section{Funding:}

National Institutes of Health grant R01 HL144131 (AKK and MDO)

National Institutes of Health grant S10OD020011 (MDO)

National Institutes of Health grant S10OD030275 (MDO)

National Institutes of Health grant T-32-GM007315 (SC)

National Institutes of Health grant R01GM080403 (JM)

National Institutes of Health grant R01HL122010 (JM)

National Institutes of Health grant R01GM129261 (JM)

Humboldt Professorship of the Alexander von Humboldt Foundation (JM)

\section{Author contributions:}

Conceptualization: AKK, MDO, EK, HSM, JM

Methodology: JCP, JC, BH, YP

Investigation: JCP, BH, YP, JC, AG, SC

Data curation: JCP, JC

Formal analysis: JCP, AG, JC, EK

Writing- original draft: AKK, MDO, EK

Writing- review and editing: JCP, BH, AG, JC, YP, SC, HSM, JM, EK, AKK, MDO

Visualization: JCP, BH, AG, SC, HSM, EK, MDO

Supervision: AKK, MDO, EK, JM

Project administration: AKK, MDO

Funding acquisition: AKK, MDO, JM.

Competing interests: The authors declare no competing interests.

Data and materials availability: The cryo-EM volume and the structure coordinates have been deposited in the Electron Microscopy Data Bank and the Protein Data Bank under accession codes EMD-XXXX and XXXX. 
bioRxiv preprint doi: https://doi.org/10.1101/2022.02.17.480763; this version posted February 17,2022 . The copyright holder for this preprint (which was not certified by peer review) is the author/funder, who has granted bioRxiv a license to display the preprint in perpetuity. It is made available under aCC-BY-NC 4.0 International license.

\section{Supplementary Materials:}

Materials and Methods

Figs. S1 to S11

Tables S1

References (37-62) 

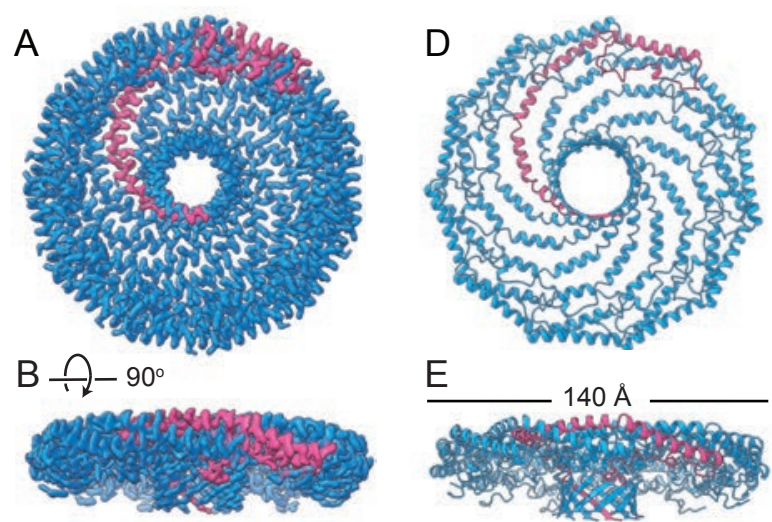

G
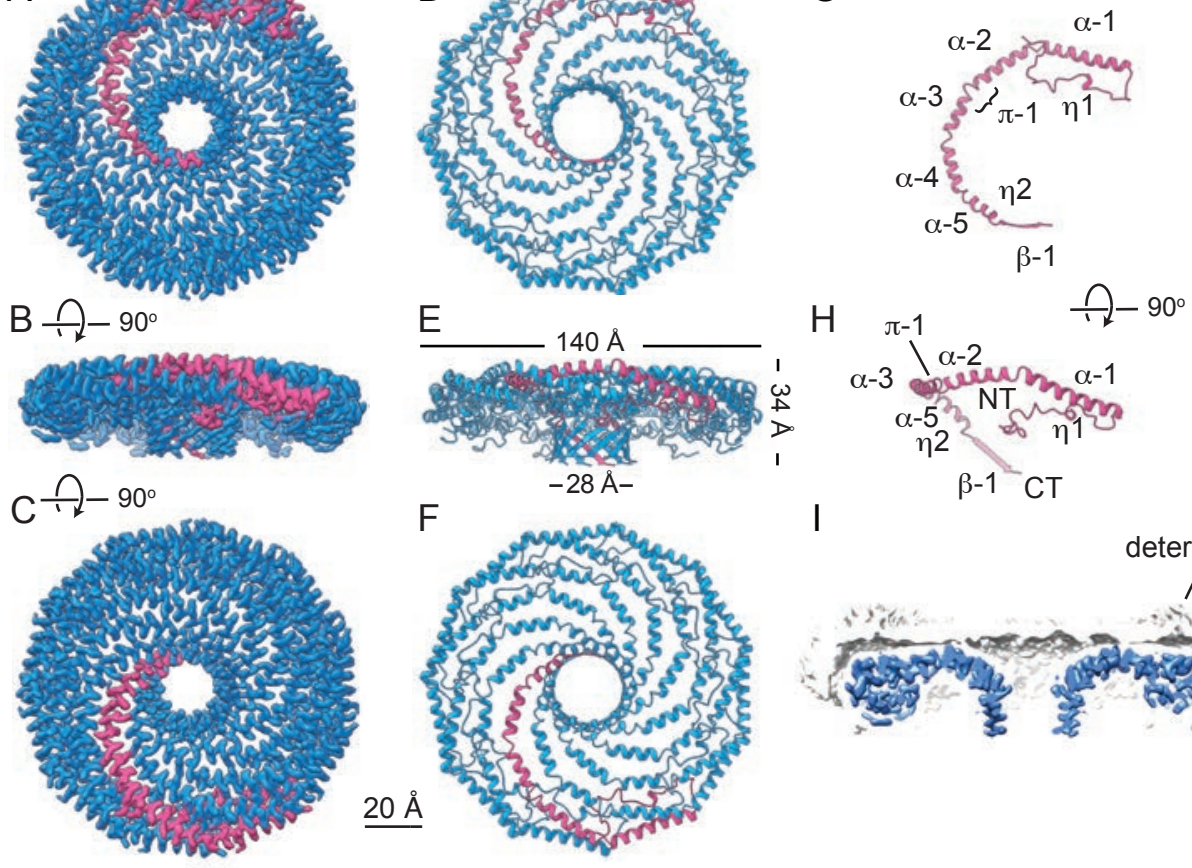

I

$$
\beta-1-C T
$$

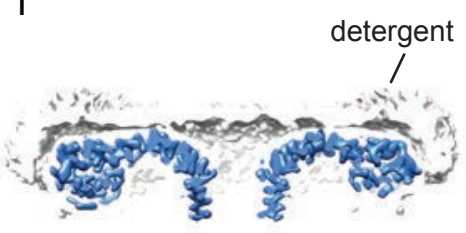

Figure 1. Cryo-EM structure of the $8 \mathrm{~S}$ Cav1 complex. (A-C) $90^{\circ}$ rotated views of the cryo-EM density of the $8 \mathrm{~S}$ Cav1 complex at $3.5 \AA$ with 11 -fold symmetry. The complex is a disc-shaped structure composed of tightly packed $\alpha$-helices and a cylindrical $\beta$-barrel. Magenta, Cav1 protomer. (D-F). Secondary structure model of the refined 8S Cav1 complex. Same views as shown in A-C. Dimensions of complex are labeled in E. (G-H) Secondary structure of Cav1 protomer with secondary structural features and position of the $\mathrm{N}$ - and $\mathrm{C}$ - termini labeled. (I) Central slice of the density map (blue) with detergent micelle (light grey). Scale bar for all panels, $20 \AA$. 

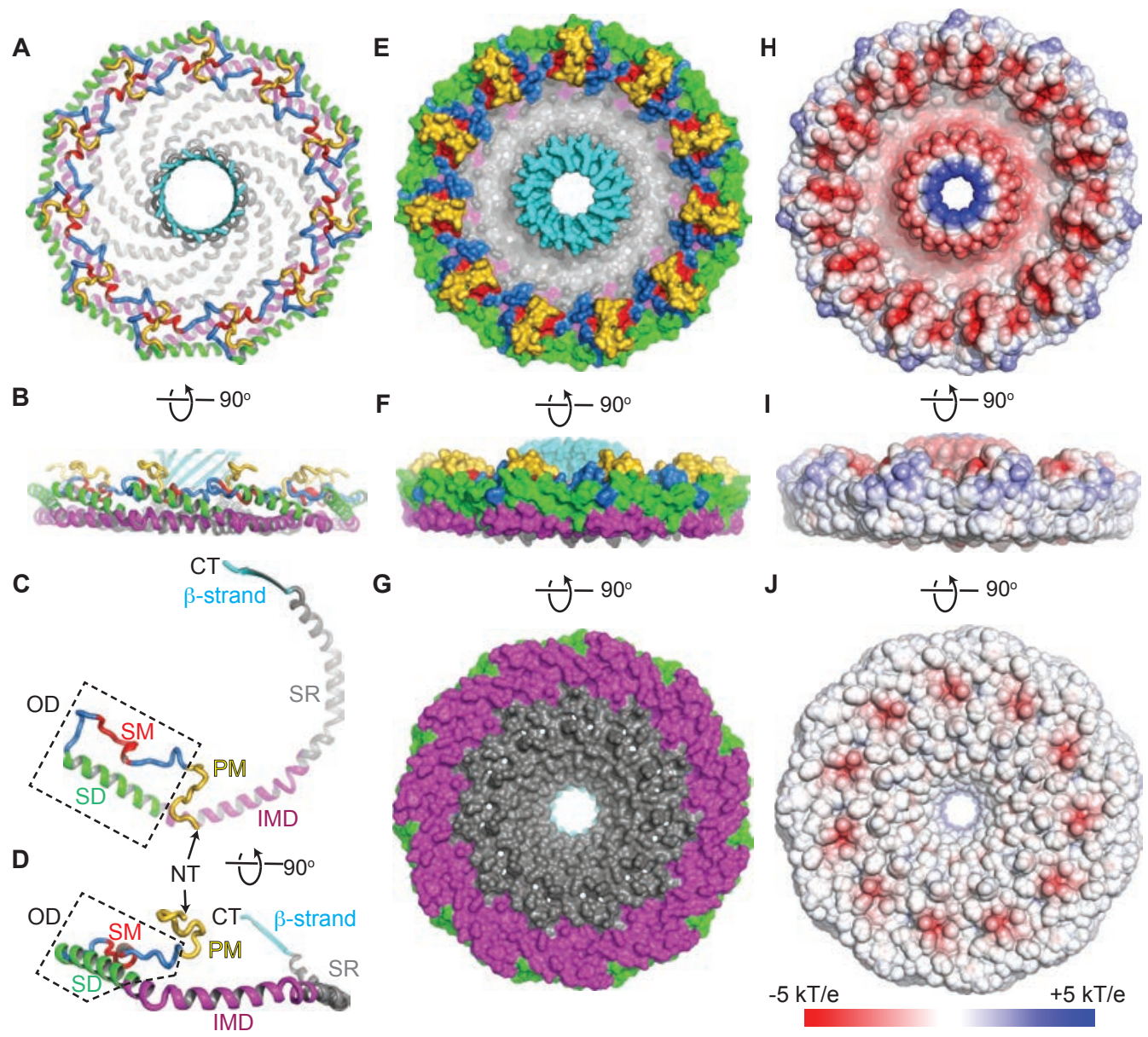

Figure 2. Structure of Cav1. (A) $3.5 \AA$ resolution structure of the $8 \mathrm{~S}$ Cav1 complex as viewed from the cytoplasmic face. (B) Structure rotated $90^{\circ}$. (C-D) Structure of Cav1 rotated $90^{\circ}$. The positions of previously defined regions are labeled: SM, signature motif (red); SD, scaffolding domain (green); and IMD, intermembrane domain (purple). The OD, which contains the SM and $\mathrm{SD}$, is indicated by the dashed box. New structurally defined motifs include: PM, pin motif (yellow), SR, spoke region (grey); and $\beta$-strand (cyan). N-terminus (NT) and C-terminus (CT) are marked with arrows. (E-G) Space filling model of the $8 \mathrm{~S}$ Cav1 complex rotated $90^{\circ}$. Color scheme identical as in A-D. (H-J) Space filling model of the 8 S Cav1 complex rotated $90^{\circ}$ showing the charge of the amino acids. Red, negative; grey, neutral; blue, positive. 

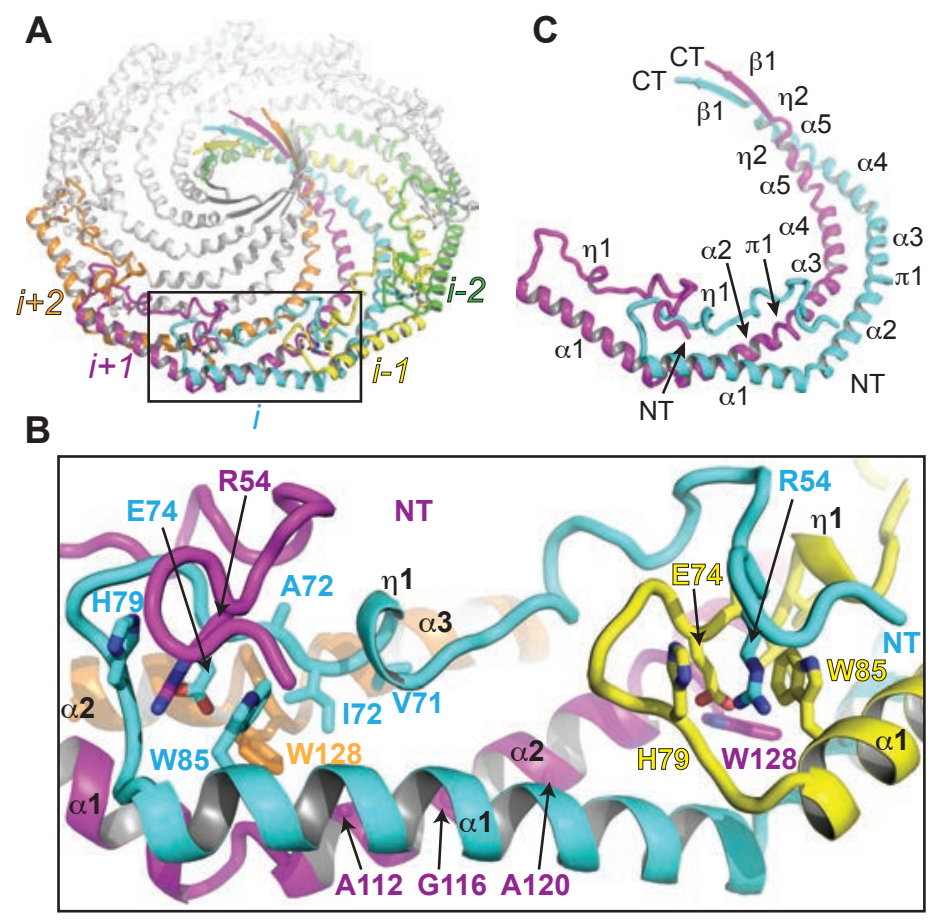

Figure 3. Cav1 $8 \mathrm{~S}$ complex is stabilized by extensive interactions along the length of the protomers. (A) Overall structure of the $8 \mathrm{~S}$ complex highlighting 5 distinctly colored protomers labeled i-2 to i+2. (B) Zoomed view of the rim region (box in panel A) highlighting key interacting residues. (C) Packing of two protomers. Secondary structure elements are labeled. 
bioRxiv preprint doi: https://doi.org/10.1101/2022.02.17.480763; this version posted February 17,2022 . The copyright holder for this preprint (which was not certified by peer review) is the author/funder, who has granted bioRxiv a license to display the preprint in perpetuity. It is made available under aCC-BY-NC 4.0 International license.

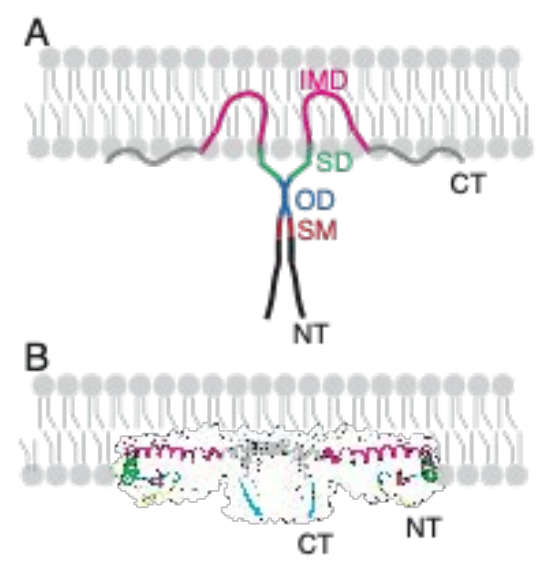

Figure 4. Proposed model for Cav1 $8 \mathrm{~S}$ complex association with membranes. (A) Classical model of Cav1 oligomer organization and membrane interaction. (B) Structure-based model. See text for details. Coloring scheme matches Figure 2A-D. 